\title{
Conscious Modulation in Normal Sleep
}

\author{
Rafael J. Salín-Pascual*
}

Full-Time Professor of Psychiatry, School of Medicine, National University Autonomous of Mexico

\begin{abstract}
The phenomenological relationship between consciousness and sleep are reviewed. Consciousness has a selfreflexive component, and while sleeping that is not working as in awakenings. The necessity of some change in narrow concepts of conscious as to be aware and self reflective as well of some aspects of Altered State of Consciousness are explained. In fact, in rapid eye movements [REM] sleep is the sleep stage in which some perception of consciousness is found self-perception in REM sleep is a common phenomenon, but to be aware of that is called lucid dreams. Also sleep paralysis produce some activation of self-awareness.
\end{abstract}

The lack of continuity between cortical areas in delta sleep stages could explain the loss of consciousness is such sleep stage, which support the idea of cortical continuity as a necessary condition for to achieve this integrative process. A question that emerges after update the knowledge about sleep and consciousness is what is the minimum degree of connectivity and activity of the central nervous system for to be conscious?

New paradigms in neurosciences like connectome and enactivation, removes reductionist approaches to the main - body issue. Enactive approach could be used as a paradigm to understand cognitive activity in three neurophysiology stages. As a proposal, it can be figured out as follows: (A) In wakefulness, the enactive phenomenon has priority to start whatever action is necessary. A biofeedback to make corrections in order to improve the motor programs, mind theory or any other active strategies; (B) In REM sleep in which there is not external stimuli. Those are generated from the sensorial nuclei in the brainstem which generates internal electrical activity that follows sensorial pathways [i.e., PGO waves] with visual and emotional targets [i.e., occipital visual cortex and amygdale complex] and probably without enactivation, but inner generating stimulation so the brain cortex is activated mainly by brainstem without any anticipatory top-down events; and (C) In delta sleep, in which there are no enactivation and no sensorial inputs, but also there is no interconnectivity in the brain cortex, which is a necessary condition for consciousness and memory.

New technical tools as functional magnetic resonance, trans-cranial magnetic stimulation, and magnetic electroencephalography have open new approaches for develop feasible hypotheses in this field.

Keywords: Sleep, Consciousness, Autoscopy, REM sleep, Dreams.

\section{INTRODUCTION}

Cognitive processes are a group of functions that are related to the brain activities, and to be conscious is part of that [1]. From clinical point of view unconsciousness is characterized by a lack of response to external stimuli, which is observed in coma and deep anesthesia. However, in slow wave sleep there is also a loss of consciousness. The brain reduces the reactivity related to both internal as well as some external information, that still reaches the brain cortex in such physiological condition, for example auditory stimuli, but without spread out or contextualizing that relevant information. The stream of information is significantly reduced in sleep; however there are oscillation of periods of unconsciousness and altered states of consciousness, like perception without external objects or hallucinations, mainly during dreams with all their manifestations [2,3]. That type of oscillation, the mechanisms behind that and the repercussion of such process that can be unconscious in neurophysiologic

*Address correspondence to this author at the Hidalgo 187 Casa 7, Barrio del Niño Jesús - Tlalpan, 14380 México City, México; Tel: [05255] 5734198; Fax: 05255-5734198:

E-mails: rafasalin@yahoo.com, rafasalinpas@gmail.com way, in overall cognitive process are part of the present review.

The neurophysiologic basis of several aspects of human consciousness has been explored using functional brain imaging techniques. For example, the brain regional mediation of some processes underlying perceptual awareness have studied in hallucinations as a model of inner brain activation [4]; subjective perceptual shifts when dissimilar images are presented to both eyes and implicit versus explicit awareness of visual stimuli [e.g., by studying patients who exhibit 'blind sight'] [5-7]. The strategy employed for to explore these functional stages like brain imaging are straightforward; scans obtained during normal conscious functioning are contrasted with those obtained during an abnormal or otherwise altered state (such as a shift in the level of perceptual awareness). Thus, functional brain imaging techniques have been used to highlight the brain regional activation/deactivation patterns that mediate even the subtlest nuance of human conscious experience.

In operative terms consciousness is an emergent property of cerebral cortex activity. However it is part of all the brain process in an integrated framework. It is elaborated and it takes the control of our activities. There are some degrees of 
difficulties in brain's investigation of consciousness. The circularity in which the brains are under self-investigation entail self-reference and bias. The thinking process cannot pass this point. Second, as an organ of integration and survival, it seems that cannot permit and easily inquiry into itself by the first person approach. The mirage of dualism is base on this two phenomenon $[1,8]$.

Self-awareness is not well understood. There are some speculations, about information and integration capabilities specifically in brain cortex. Their events are time related. Awareness requires 400 to 500 milliseconds for processing. However, the reflective awareness it seems a specific human characteristics $[9,10]$. There are few clues about how it is organized. The human capacity for experiencing own conscious in a self-ascribed manner can break down and become disordered and distorted in some sleep stages as well as sleep transitions. However, that is not the only example of how cognitive process are imprecise in sleep [8]. Self-perception in REM [rapid eye movement] sleep is a common phenomenon, but to be aware of that is called lucid dreams. Also sleep paralysis produce some activation of self-awareness [11-13].

New tools as brain imaging, high density EEG, among others allowed some clarifications about neurological process [14-16]. Consciousness during sleep and even autoscopy and lack of awareness in dissociative behaviors related to sleep and epilepsy, were the subject in the literature fiction, back in XIX century like some writers as Guy de Maupassant in the short story called "La Horla" [17].

The main goal of this article is to describe some elements in the field of sleep and cognition and the modulation of neural function that results in altered states of consciousness during the different sleep stages. The self-perception in sleep, the lack of reflective awareness; autoscopy, out of the body perception, intentionality, and some altered states of consciousness, which differentiate sleep from wakefulness, are presented here and to propose and hypothesis about the functions that all these process accomplish both in sleep and wakefulness.

\section{DREAM AS ALTERED STATES OF CONSCIOUS- NESS}

Dreams, as we remember when we are awake, are collections of memories and sensorial inner activation without external objects and also a cinematographic type of edition of several dreams each night, mix with relevant memories and emotions that we experienced when fully awake. Dreams resembles about the same situation that happens when people are in full awake. However, cognitive process are qualitative different. Dreaming and perhaps other mental activity that we experienced while sleeping are not full consciousness, but altered state of consciousness, without self-awareness. When awake, beyond sleep inertia, we remember some particular dreams, those with special meaning [2].

Altered State of Consciousness (ASC) are common experienced in our everyday life. That may result like mental confusion that may be observed in different clinical conditions: drunkenness, delirious state with fever, under the influence of some drugs like marijuana, psilocybin or mescaline. ASC has been defined as a qualitative alteration in the overall pattern of mental functioning; different from the one was used in the everyday activities when awake. ASC is radical different from the perspective of brain functioning. Fragmentation or non-continuity of the experiences, misperception, bizarreness and lack of recollection of what happens at each particular moment (memory about that are fragmented and not in context) $[18,3,19,20,21]$.

\section{MENTAL ACTIVITY AND SLEEP}

Cognitive processes have been evaluated in the fullconscious state. Some differences in cognitive functions could be detected through the multiple stages of vigilance (I.e., active and passive wakefulness; hypnosis and meditation), as well in sleep with slow wave sleep (also called nonREM sleep) and REM sleep. This variation in cognition in the different stages of vigilance could be related to the mechanism of regulation of those stages and also differences in the levels of integration and functions $[22,23]$.

Mental activity is changing during sleep. In REM sleep, there is a type of psychotic way of thinking; there are some transgression to the laws of physics and logic. For instance, people are flying, or with relatives that already have passed away. They may fell down from a high altitude and land easily, or trespass walls without any harm. For the dreamer, at that moment of that experienced, they believe that what just happens is real. Studies with fMRI show a light up of all cortexes, more at the occipital areas and less in frontal lobes [24].

Nevertheless, the mental activity during Non-REM sleep is quite different, more linear and fragmented, like an obsessive way of thinking. An interesting study shows us that some of this activity could be explained by the concept of modular cortex. They are anatomical and functional patches of active and inactive zones of brain cortex, when in slow wave sleep. These on and off, as a way of functioning suggest that consciousness depends not much on firing rates, synchronization at specific frequency bands, or even sensorial inputs per_se. It depends more on the brain's ability to integrate information, which is contingent on the effective connection between functional and specialized regions within the cortex and the thalamic and sub-cortical systems [24]. Effective connectivity is related to the ability of a set of circuits to function in a cause-effect condition, modifying by that the activity of other group of neurons in a cross-talk way of communication [25]. The fading out of consciousness during Non-REM sleep episodes at the beginning of the night, is evidenced by unawareness or blank reports of cognitive activity upon awakening [26, 27]. That could be associated with an impairment of cortical effective connectivity. To test such idea, Tononi and his team [28-30] recorded electrical activity in brains of six volunteers using highdensity EEG. Before the subjects nodded off, the researchers stimulated a small patch of right frontal cortex with transcraneal magnetic stimulation [TMS] [30], a noninvasive method that uses magnetic pulses to induce an electric current inside the brain. The EEG records revealed how the neural activity triggered by TMS spread from the site of stimulation to other parts of the cortex. The team repeated the same experiment once the subjects had entered non-rapid eye movement [nonREM] sleep. Noise-canceling earphones ensured that subjects could not detect the sound of the TMS magnet. When the subjects were awake, TMS elicited waves of neural activ- 
ity that spread through neighboring areas of the right frontal and parietal cortex and to corresponding regions on the left side of the brain. During non-REM sleep, the same TMS stimulus elicited neural activity at the site of stimulation, without spreading beyond that point. These findings suggest that different areas of cortex indeed stop talking to each other during non-REM sleep-a stage of sleep in which people often report little awareness and no conscious experience.

Also this finding explains why it is difficult to remember what is performed in that sleep stages, as happens in nocturnal terror, somnambulism and even in automatism in sleep deprived subjects. This is the only period in which we are unconscious, in some relative way, because density of delta waves is not uniform [in fact, there is the distinction between sleep stage III and IV in humans]. The brain became functioning in modules during that sleep phase. Non-REM sleep has more diencephalon type of control [anterior hypothalamus and intralaminar thalamic unspecific nuclei] - that elicit such cortical and synchronic activity; while REM sleep is more brain stems regulated, with specific thalamic nuclei [i.e., external geniculate bodies], that are targeting some posterior area of the cortex [31-34]. There are the possibilities that in some persons with conscious in REM sleep and even with autoscopy, that some areas of frontal lobe, like dorsolateral prefrontal cortex, were more active and then allow that without awakening the subjects they were aware of to be in a dream $[35,36]$.

\section{THE SENSATION - PERCEPTION INTEGRATION}

There is evidence that some areas of the brain are active ahead whichever behavior follows, that could be observed whiting the mere act of thinking about doing something, that has been named Intentionality, and that has been studied scientifically since the discovery of the slow negative electroencephalographic activity, continuing self-initiated movement $[37,38]$ and that is important for self perception. Awareness is not equivalent to be consciousness, but one must be conscious for to be aware of us. It can be said that a person could be conscious but without awareness, but always awareness has to be in a full conscious state, although there are some conditions in which self-awareness could happen during REM sleep, and in the transition between sleep state and awake, that is not always the case when a person is deeply asleep.

Self-awareness can be defined as to be self-being aware of one [39].

Also it is like planning in advance or kind of navigation devices $[39,40]$. Prediction about what follows after different events are one of the main cognitive functions, and has all the adaptation display to attack or avoid the enemy. Part of the neurobiology of that command should be at the brainstem level. The planning of motor but also non-motor activities are the main functions of the brain, which is observed at both levels at the conscious level as well as unconsciousness. One of the functions of dreaming could be related to that in model of virtual reality.

\section{NEURAL CIRCUITS OF SELF-AWARENESS}

The self-awareness is a complex process because it can have many sources [40]. Researchers in the area have proposed the following sets of self-representation: Protoself;
Emotional Self; Spatial Self; Automatic Self; Appetitive Self; Social Self; It Remembered Self and Neocortical Observing Self. A different set of circuits some of them interconnected, are part of these self-represented areas: Thalamus, cortex, hypothalamus, basal ganglia, amygdale, and monoaminergic nuclei in the brain stem among others [40, 41].

It has been mentioned that perception is not a passive process, and that is an aspects of the reception of all these signals [Primary cortex areas], but also because the organisms participate with movements to focus de signal. All these aspects of modulation of sensorial and motor functions are active process that have named Enactivation (Top-down process) [42].

Enactive approach could be used as a paradigm to understand cognitive activity in three neurophysiology stages. As a proposal, it can be figured out as follows: (A) In wakefulness, the enactive phenomenon has priority to start whatever action is necessary. A biofeedback to make corrections in order to improve the motor programs, mind theory or any other active strategies; (B) In REM sleep in which there is not external stimuli. Those are generated from the sensorial nuclei in the brainstem which generates internal electrical activity that follows sensorial pathways (i.e., PGO waves) with visual and emotional targets (i.e., occipital visual cortex and amygdale complex) and probably without enactivation, but inner generating stimulation so the brain cortex is activated mainly by brainstem without any anticipatory topdown events; and [C] In delta sleep, in which there are no enactivation and no sensorial inputs, but also there is no interconnectivity in the brain cortex, which is a necessary condition for consciousness and memory [brain deals with memory on contextual models not in postal like paradigm, as computers do], because there is no contextual connectivity [42].

During awake time, sensorial information that reach the bran cortex divert, one set of information goes to the primary an associative areas and then to regions that control the particular areas of the body that we need for movement, another goes to regions, which are in charge of surveillance of the movements. If the actual sensation that is received does not match our prediction, our brain becomes aware of the difference, and at least two things can happen: go on and creating by that something as a myth or to establish a research protocol, by questioning some of the basic premises of the phenomenon that was observed [44].

The sight of someone being poked, for example, stimulates neurons in the pain region of our own brains. Blakemore and her colleagues [43, 44] have found that even seeing someone be touched can start mirror neurons. They showed a group of volunteers some videos of other people being touched on the left or right side of their face or neck. The videos elicited the same responses in some areas of the volunteers' brains as occurred when the volunteers were touched on the corresponding parts of their own bodies.

Experimental blindness has used as a model for to study enactivation, because it shows that, even if you have a full sensorial pathway [i.e., retina, optic nerve, visual tracts and occipital cortex], that is not enough if you do not have the previous experiences, which could put everything in a coherent context [44]. 
Experimental blind people had some defect in the crystalline when they were born [congenital cataracts], that is a congenital problem in both eyes, which result in blindness. However, after removing cataracts, patients still have some trouble, because they do not have previous visual perception that may develop the contextualization of that incoming experience [42].

Are we aware of initiating movement? Being aware of initiating and controlling actions is a major component of conscious experience, but many aspects of action occur without our awareness. Evidence that sensations are associated with actual movements is unavailable to the awareness. That comes from a study in which the sensory consequences of movement were made to deviate from a subject's anticipation. Sensory prediction might underlie the ability to prepare and imagine movements. It is well established that imagining a movement and preparing to move start in a subset of brain regions that are also made active by executing a movement [44-49]. Healthy subjects may confuse actions performed in the imagination and in reality when they are asked to recall actions two weeks later. In a recent functional magnetic resonance imaging [fMRI] study, subjects were scanned while they executed movements or imagined making the same movements. A direct comparison between brain activity during imagined and executed movements revealed that imagining movements starts at the left posterior and inferior parietal lobe largely than when executing the same movement in reality.

\section{CONSCIOUS AND SLEEP}

A great percentage of sensory stimuli do not reach conscious perception during sleep. It has been thought that the thalamus prevents the relay of sensory information to cortex during sleep, but the consequences for cortical responses to sensory signals in this physiological state remain unclear $[50,51]$. The recording from two auditory cortical areas downstream of the thalamus in naturally sleeping in marmoset monkeys was used as a model for to test the above statement. Single neurons in primary auditory cortex either increased or decreased their responses during sleep compared with wakefulness. In secondary auditory cortical area, the response modulation was also bidirectional and showed no clear systematic depressive effect of sleep. When averaged across neurons, sound-evoked activity in these two auditory cortical areas was surprisingly well preserved during sleep. Neural responses to acoustic stimulation were present during both slow-wave and rapid-eye movement sleep, and were repeatedly observed over multiple sleep cycles, and demonstrated similar discharge patterns to the responses recorded during wakefulness in the same neuron. These results suggest that the thalamus is not as effective gate for the flow of sensory information as previously thought. At the cortical level, a novel pattern of activation/deactivation appears across neurons. Because the neural signal reaches as far as secondary auditory cortex, this leaves open the possibility of altered sensory processing of auditory information during sleep [52].

REM sleep is one of the different phases of sleep. Because mental activity changes in some aspects in this sleep stage, although the basic process of wakefulness still remains the same. Whereas traditional sleep was hypotheses to be regulated through top-down control mechanisms, recent approaches have emphasized that sleep is emerging and regulated in a use-dependent [homeostatic] manner. Traditional markers of sleep homeostasis, as the electroencephalogram slow-wave activity, have been linked to changes in connectivity and plasticity in local neuronal networks. Thus waking experience-induced local network changes may be sensed by the sleep homeostatic process and used to mediate sleepdependent events, benefiting network stabilization and memory consolidation. Although many questions remain unanswered, the available data suggests that sleep function will be best understood by an analysis that integrates sleep's many functional levels with its local homeostatic regulation $[52,53]$.

The burst of PGO waves started in the cat, a few seconds before REM sleep started in the EEG, and this phenomenon lasted during all the time those animals are in REM sleep. Researchers in this field separated REM sleep phenomena in two sets of elements: Tonic and phasic events. Tonic variables are without noticeable variation during the full REM sleep duration time. EEG desynchronized, penile erection and loss of muscular tone of the antigravity muscles. Phasic events are on and off kind of activities, like trend of rapid eye movements, myoclonic contractions [facial and limbs muscles] changes in respiration frequency, hearth rate, blood pressure [54-57],

As has been mentioned before, there is some evidence that neural communication between brain cortexes breaks down during delta sleep and that may account for the loose of consciousness [58]. That does not infer that the brain is completely turned off during deep delta sleep. The activity of the cortical network that remain active may be higher as has been demonstrated by Laufs et al. [59], they examined blood oxygen dependent [BOLD] signal functional connectivity. When subjects were at fMRI. Dorsal network activity increased during light sleep comparing with wakefulness. The areas that explored were the dorsal attention system; the executive control system and a default system [a set of regions that are suppressed relatively to wakefulness]. In addition, they examined with fMRI three primary sensory systems [visual, auditory, and motor]. The results support a functional disconnection in Non-REM sleep [60-62].

The idea about how the cortex works is based on information theory, in which the cortex is reacting only after the sensorial stimuli, arrives to the primary areas. However, that it is not longer sustained [reflexological theory, cortex as a passive cinema screen]. Sleep in fact, is the most excellent example of self-organized operations within neural circuits as a complex system. Brain "state" has macroscopic variables, which are observed in oscillatory modes. Each particular state is generated by the participating neurons and defined by a set of parameters such as neurotransmitters, voltagegated ion channels, activation of receptors and other circuit's actions. That is more like a choreographic activity that at the end determines a complex behavior.

Sleep represents a natural model for study brain functions without external stimuli or important reduction of them; it was found that sleep is a well-organized behavior with heterogeneous stages. The interaction between Thalamus, brainstem and neocortex, are good example of that [51]. 
Other than the well-known EEG activities, there was found a slow oscillation frequency. That was discovered from three different groups. Steriade et al., in 1993 published a novel oscillatory cortical pattern and they named "slow" oscillation [63-70]. They report that slow frequency was less than a second. This is also called the hyper polarized state. Cortical slow oscillation can trigger thalamic-cortical sleep spindles. The mechanisms responsible for that take to the active network back to silence, and are not well understood. Reducing increase resistance of neurons, active-dependent $\mathrm{K}+$ currents and gain inhibitory mechanisms are taken collectively as the explanation of such phenomenon. Anesthetics that increase $\mathrm{K}+$ conductance or potentiated the action of GABA can prolonged the down oscillation state.

The longest silent period last between 100 to 500 milliseconds during stage 3 and 4 of sleep. That is associated with delta sleep [EEG frequency of 0.5 to 1 per second and with 70 to 90 micro volts]. That phenomenon is observed in all areas of the cortex, with a flip-flop behavior of cortical modules. Also it is a reflex of up and down interaction of thalamus and cortex $[64,65]$. The mechanism of so called "K complex" in EEG during early sleep stages, has explained as some activation of sense-perceptive area, because it is generated in that cortex areas.

Complex systems with a predictable "path" in space and in a period of time are called deterministic. The causal interaction between thalamus and neo-cortex are like that. During sleep, cortical neuronal networks oscillate slowly $(<1 \mathrm{~Hz})$ between periods of activity (UP states) and silence (DOWN states). UP states favor the interaction between thalamic generated spindles [7-14 Hz] and cortically generated gamma [30-80 Hz] waves. So brain cortex, still active in delta sleep but without consciousness. From these evidences it can be said that base on enactivation process, sleep and wake stages could be organized in three: wakefulness with enactivation process (Top down), in which sensorial information acts in a model of null hypothesis; Non-REM sleep, in which there are not enactivation (cortex modules are not interconnected), and not sensorial information reaching the brain; and REM sleep where input information from brainstem are reaching some specific thalamic and cortical areas, without enactivation.

\section{WHAT IS THE MINIMUM NEURAL ACTIVITY NECESSARY TO ACHIEVE CONSCIOUSNESS?}

Paradigms are models that could have operative validation to explain some aspects of reality. Neurons and synapse have a limitation for those goals, because among other things they are not autonomous because they keep interacting each other in circuits with perfectible functions. Giulio Tononi and Gerald M. Edelman [71] looked for the minimum elements of a conscious experience, emphasizing two properties: conscious experience is integrated [each conscious scene is unified] and at the same time differentiated [within a short period of time, one can experience any of the immense number of different conscious states]. Then, they consider the neurobiological data indicating that neural processes are associated with conscious experience that are integrated and differentiated; and provide tools for measuring that [called functional clustering] and differentiation (called neural complexity). Those are applicable to actual neural processes.
This leads us to formulate, an operational criteria for determining whether the activity of a group of neurons contributes to conscious experience. These criteria are incorporated into the dynamic core hypothesis, a testable proposal concerning the neural substrate of conscious experience.

A fundamental aspect of the stream of consciousness is that it is unified in a holistic way. Integration is a property shared by every conscious experience irrespective of its specific content: Each conscious state integrate single "scene" that cannot be decomposed into independent components. Integration is best appreciated by considering the impossibility of conceiving a conscious scene that is not integrated. Unity also entails that conscious experience is private, that is, it is always experienced from a particular point of view in each subject and cannot be shared [1].

While each conscious state is complete integrated; perhaps the most remarkable property of conscious experience is its extraordinary differentiation or complexity. The number of different conscious states that can be accessed over a short period of time is large. For example, even if we consider visual images, we can easy discriminate innumerable scenes within a fraction of a second.

Changes in specific aspects of conscious experience correlate with changes in activity in specific brain areas, whether the experience is driven by external stimuli, by memory, or by imagery and dreams. Tononi and Edelman [71] suggested that a key neural mechanism underlying conscious experience is the interactions between the posterior thalami-cortical areas involved in perceptual categorization and anterior areas related to memory, value, and planning for action. Interactions among neuronal groups in several brain areas may be necessary to generate a unified neural process corresponding to a multimodal conscious scene.

The degree of activation and deactivation of thalamus and cortical circuits is highly correlated with the stages of vigilance. In subjects who are comatose or deeply anesthetized, unconsciousness is associated with a profound depression of neural activity in both the cerebral cortex and thalamus [13]. During slow-wave sleep, in which consciousness is lost, cerebral blood flow is global reduced as compared to waking and REM (rapid eye movement) sleep [14].

\section{BRAIN RHYTHMS AND CONSCIOUSNESS}

Two rhythms that show particularly strong behavioral correlation are the $4-8 \mathrm{~Hz}$ theta rhythm and the $30-80 \mathrm{~Hz}$ gamma rhythm $[72,73]$. The former rhythm has been studied extensively in rodents, in which it is especially prominent in the hippocampus during locomotion, orienting, and other voluntary behaviors. The higher-frequency of gamma rhythm has been linked recently to increased hemodynamic activity [as measured using functional magnetic resonance imaging] in cat, monkey, and human. Recent analyses of intracranial electroencephalograph recordings, taken from neurosurgical patients, have provided evidence for locally generated brain oscillations in human hippocampus and neocortex. Along with scalp EEG and magnetoencephalographic [MEG] recordings, these studies reveal evidence that synchronous oscillations in the theta- and gamma-frequency bands are modulated by behavioral and task variables. The convergence of recent evidence suggests 
that brain oscillations are generated in almost every part of the brain and they play a wide range of functions in both human and animal cognition [72].

Gamma oscillation is one of the EEG rhythms that most characterized the pattern of waking. Activated cortex is equivalent to gamma oscillation. Sensory perturbations can easily riser gamma rhythm in the wake state, whereas the same stimulus is largely unaffected during REM sleep. This is a relevant difference in function between the two neurophysiologic stages, that although similar EEG patterns have different reactivity to external stimuli. Gamma oscillation depends on GABA-A receptors decay or shutting. These Gamma rhythms represent an explanation for the "binding problem". The characteristics of a single object that are analyzed in different areas of the brain but reconstructed in less than 200 milliseconds with all the object's characteristics [color, form, high, structure, smell, taste, and more] [73].

By applying fMRI and magnetic resonance spectroscopic imaging [MRS] at the same time, to nonhuman primate, the most frequently used laboratory animal for study the neural basis of cognition, scientists may be able to investigate levels of neural organization that cannot be studied by electrodes alone. These include [a] long-range interactions between different brain structures, [b] task- and learning-related neurochemical changes by means of localized in vivo spectroscopy or MRS, [c] dynamic connectivity patterns by means of labeling techniques involving MR contrast agents, and [d] plasticity and reorganization after experimentally placed focal lesions.

The fMRI technique, like most current brain imaging techniques, capitalizes on the coupling of cerebral blood flow (CBF), energy demand, and neural activity. The interactions between these variables are overwhelmingly complex and involve unified factors such as the type of neural activity involved, the cell groups generating this activity, the link between this activity and energy demands, and the processes ultimately coupling the energy demand to the supply of energy to the brain. In this review I will concentrate on only one aspect of this complex issue, namely the type of neural activity that could play a dominant role in the generation of one sort of fMRI signal: the imaging signal capitalizing on the blood oxygen level-dependent (BOLD) contrast mechanism.

BOLD was first described by Seiji Ogawa [74] in rat brain studies with strong magnetic fields [7 and $8.4 \mathrm{~T}$ ]. He noticed that the contrast of very high resolution brain images [65 -65 - $700 \mathrm{~m} 3$ ] acquired with a gradient-echo pulse sequence depicts a number of dark lines of varying thickness that could not be seen when the usual spin echo sequences were used. They turned out to be signal dropouts from blood vessels [74]. In other words, by accentuating the susceptibility effects of deoxyhemoglobin in the venous blood with gradient-echo techniques, Ogawa discovered a contrast mechanism reflecting the blood oxygen level. With remarkable insight he realized the potential importance of its application, concluding that BOLD contrast adds an additional feature to magnetic resonance imaging and complements other techniques that are attempting to provide positron emission tomography-like measurements related to regional neural activity".
Using above technological approach, Kauffman et al [75], have found that several brain regions activity was reduced during all Non-REM sleep stages. First, most of these deactivated areas are located in the frontal lobes [inferior frontal, middle frontal, superior frontal, medial frontal, precentral gyrus and paracentral lobule] with a predominance in the right cerebrum. Secondly, regions of the limbic lobe such as the anterior cingulate cortex and precentral gyrus were also less activated during Non-REM sleep. Furthermore, the anterior nucleus of the thalamus and the body of the caudate nucleus showed reduced activity, again with predominance in the right hemisphere. Thirdly, temporal (superior temporal gyrus), parietal [post-central gyrus, inferior parietal lobule], occipital (cuneus, precuneus) as well as insular activation [restricted to the right anterior Brodmann area [BA] 13] diminished during all Non-REM sleep [75].

In REM sleep the level of energy metabolism is similar to waking level, with a different distribution. Areas that are responsible for REM sleep are lighting up, like pontine tegmentum and specific thalamic nuclei. However some emotion-involved areas are active too. For instance limbic and paralimbic structures. Also temporo-occipital areas, where the primary PGOs are targeting. In contrast there are a relative less activation of frontal and parietal regions. Functional interaction between cortex and striatum is reduced contrary of what happens in full awakening, while the activity of the amygdale and temporo-occipital areas are higher than wakefulness. This activation and deactivation of brain areas explain some of the characteristics of dream in REM sleep [75]. Emotional aspects and daily activities, but without a context, changing scenarios and even subjects. That supports the lack on enactivation of top down mechanisms.

Thus, dreams are a mix of visual and auditory hallucinations and thought to go along with the process of activating brain circuits for the purposes of consolidating very recent memories. Because synapses are subject continuously to depletion processes, if they were not periodically refreshed during sleep, many memories would be lost. The contents of the dreams that occur when ensembles of memory circuit are reinforced by thought to be derived from the memories stored in the circuits, some of which may be combined in incongruous or bizarre ways.

\section{ARE WE INSIDE OF OUR BRAIN?}

Self-perception is a heuristic model in which the past and present experiences collide. It is built up through early years of development, so there are biological and social experiences put there together. That representation has been localized in the temporo-parieto-occipital areas, from de right side. There are some experiments as well as experimental manipulation that provide some empirical information about how to deceiving the brain.

Recently, two research groups have manipulated selflocalization by filming human subjects from behind their back and relaying the captured images in real-time to stereoscopic goggles worn by the participants. Thus the subjects saw a virtual copy of their body in front of themselves. In the study by Lenggenhager and colleagues [76], the experimenters stroked the back of their subjects so that they could see the application of this tactile stimulation on the virtual body. Participants reported feeling the strokes at the location at 
which they saw them being applied to the virtual body, rather than to their real body, and they indicated that it seemed as if the virtual body was their own. In the study by Ehrsson [77], on the other hand, the author stroked the chest of his subjects, invisibly to them, and applied synchronous strokes to a location just below the camera standing behind the participants [the rod approached the lens and then disappeared just below it with each stroke]. In this situation, the subjects reported feeling as if the rod approaching the camera touched their real chest; they said they had the impression of being located at the camera's position, and that seeing their virtual body from behind was almost like looking at someone else. These two experiments produced a misperception that led a kind of autoscopy.

That kind of phenomenon can be produced also by brain manipulation in neurosurgical procedures. An out-of-body experience $[\mathrm{OBE}]$ was repeatedly elicited during stimulation of the posterior part of the superior temporal gyrus on the right side in a patient in whom electrodes had been implanted to suppress tinnitus. Positron-emission tomographic scanning showed brain activation at the temporoparietal junction - more specifically, at the angular-supramarginal gyrus junction and the superior temporal gyrus-sulcus on the right side. Activation was also noted at the right precuneus and posterior thalamus, extending into the superior vermis. We suggest that activation of these regions is the neural correlate of the disembodiment that is part of the out-of-body experience [78].

Why autoscopy and OBE are relevant for conscience experience in sleep? If there are self-perception areas, that give us identity, this could be "the reader" of reflective awareness and perhaps also in lucid dreams.

\section{LUCID DREAMS}

A lucid dream is the one in which the person is aware that is dreaming. About "Lucid dreams", it has said that two aspects of consciousness should be first considered: consciousness as awareness (phenomenological meaning) and consciousness as strategic control (functional meaning). About awareness, three types can be distinguished: (a) Awareness as the phenomenal experiences of objects and events; (b) Awareness as meta-awareness, i.e., the awareness of mental life itself; (c) Awareness as self-awareness, i.e., the awareness of being oneself. While phenomenal experience and self-awareness are usually present during dreaming [even if many modifications are possible], meta-awareness is usually absent [apart from some particular experiences of self-reflective], with the exception of lucid dreamers. Consciousness as strategic control may also be present in dreams. The functioning of consciousness is then analyzed, following a cognitive model of dream production. In models in which dreams are supposed to be the product of the interaction of three components: (a) the bottom-up activation of mnemonic elements; (b) interpretative and elaborative topdown processes, and (c) monitoring of phenomenal experience. A feedback circulation is started among the components, where the top down interpretative organization and the conscious monitoring of the dream scene felicitates other mnemonic contents, according to the requirements of the dream plot. This dream productive activity is submitted to unconscious and conscious processes $[35,36,80]$.
To be aware of dreaming could mean some degree of prefrontal activation, preserving sleep continuity. Activity in the prefrontal cortex may distinguish the meta-awareness experienced during lucid dreams from its absence in normal dreams. To examine a possible relationship between dream lucidity and prefrontal task performance, a study was carried with a prospective 28 high school students. Participants performed the Wisconsin Card Sort and of evaluated Iowa Gambling tasks, then for 1 week kept dream journals and reported sleep quality and lucidity-related dream characteristics. Participants who exhibited a greater degree of lucidity performed significantly better on the task that engages the ventromedial prefrontal cortex [the Iowa Gambling Task], but degree of lucidity achieved did not distinguish performance on the task that engages the dorsolateral prefrontal cortex [the Wisconsin Card Sort Task], nor did it distinguish self-reported sleep quality or baseline characteristics. The association between performance on the Iowa Gambling Task and lucidity suggests a connection between lucid dreaming and ventromedial prefrontal function [79].

\section{AUTOSCOPY}

That is the experience of seeing one's body projected into external space, usually in front of oneself for a short period of time. An out-of-body experience [OBE] may be defined as the experience in which a person seems to be awake and to see his body out the world from a location outside the physical body. A closely related experience is autoscopy, which is characterized by the experience of seeing one's body in extra personal space. Both experiences are classified as autoscopy phenomenon [81, 82]. The subtle difference between them is the disembodiment sensation.

Techniques that are used to induce OBEs propose that the subjects use a supine and relaxed position [43, 44] suggesting an influence of proprioceptive, vestibular and tactile mechanisms on that event. Autoscopy and dream recall has been studied in epilepsy. The frequency of dream recall in patients with brain diseases has indicated the relationship between sleep disturbances and the functioning of cognitive processes during sleep. The ability to recall dreams was established in nearly all patients with either complex partial seizures or generalized seizures. Dream recall frequency resulted significantly higher [about twice] in patients with complex partial seizures, regardless of (a) the side of the epileptic focus, (b) the presence of a cerebral lesion detectable on a computed tomography scan, or (c) the occurrence of seizures in the previous day. The high dream recall frequency observed in these patients, regardless of the side of the epileptic focus, is in agreement with the assumption that tempo-parietal areas of both hemispheres are involved in the production and recall of the dream experience [81].

Early studies were essentially successful in describing the variations of the global level of cerebral metabolism. More recently, regional distribution of cerebral blood flow was reported. The results suggest that the permissive and executive processes of slow wave sleep and REM sleep are similar in humans and animals. They also show cortical blood flow distributions specific to each sleep stage. One of the questions that results provocative about Autoscopy and "lucid dreams", during REM sleep and wake - sleep transition is the probable activation of the prefrontal cortex in these subjects, these phenomena aloud an internal self-awareness [34]. 
The cellular mechanisms underlying the involvement of these cortical areas in sleep are not yet precisely known. They should be explored in future investigations in animals. Forthcoming research in functional neuroimaging will attempt to explore functional and effective connectivity between cerebral areas involved in sleep processes.

In humans it has been found occipital activation and frontal deactivation during REM sleep [24]. Thus, fMRI now seems to be applicable to sleep too, which should provide sleep researchers with a new method for investigating in vivo sleep physiology and pathology. This kind of work gives us an idea that during REM sleep a functional disconnection between prefrontal lobes and occipital lobes may happen. Early studies show reliable increases in function in the limbic and anterior paralimbic cortex in rapid eye movement [REM] sleep and decreases in function in higher-order cortical regions in known thalamus-cortical networks during nonREM sleep [83].

Daytime autoscopy is more a neuropsychiatric phenomena. Denning and Berrios [36], reported several cases with autoscopy. There were 38 men and 18 women, with a mean age of 39.5 years [range 13-78]. Of the subjects, 33 [59\%] had a neurological illness, most frequently epilepsy [18 cases]. Right and left sided lesions were equally represented. Psychiatric disorder was often present [33 cases, 59\%], most commonly delirium, depression or psychosis [82, 83. 84]. They were awakened during rapid eye movement sleep [REM] and asked to recall their dream. By way of coherence analyses, writings showed that rhinal-hippocampal connectivity values that were approximately twice as large for patients with good dream recall versus those patients with poor dream recall. This suggests that rhinal-hippocampal connectivity is a key factor in determining declarative memory formation [84].

Hallucination during sleep paralysis is common. The hallucinatory phenomena was distributed in three dimensions as well as distance and dispersion measures were assessed in 279 subjects for two general categories of hallucinations: Intruder hallucinations--including the presence, visual, and auditory hallucinations; and Vestibular-Motor [V-M] hallucinations--including, floating, flying, illusory motor movements, out-of-body experiences [OBEs], and autoscopy. The differences in the spatial characteristics of Intruder and V-M hallucinations largely corroborated predictions based on the respective hypothesized neural substrates of a threat started vigilance system and a bodily self neuron-matrix [84].

OBE is defined by the presence of three phenomenological characteristics: (i) disembodiment is the location of the self outside one's body]; (ii)the impression of seeing the world from an elevated and distanced visual-spatial perspective [extracorporeal, but egocentric visual-spatial perspective]; and (iii) the impression of seeing one's own body [Autoscopy] from this perspective [43, 44]. Autoscopy is part of OBE, but it is not necessarily the same thing. This may lead to the experience of seeing one's body [Autoscopy] in a position [i.e., on a bed] that does not coincide with the felt position of a one's body (i.e., under the ceiling; see above quoted example from Irwin, 1985). As OBEs are also characterized by disembodiment and elevated visual and spatial perspective, it is speculated that an additional vestibular dysfunction is present in OBEs. They suggested that OBEs are related to an integration failure of proprioceptive, tactile, and visual information regarding one's own body [disintegration in Personal space] and to a vestibular dysfunction leading to an additional disintegration between corporeal [vestibular] space and extracorporeal [visual] space.

\section{FUNCTIONAL ASPECTS OF DREAMING}

The nature of the dream-state is highly subjective and a truly personal experience making the scientific analysis of dreaming somewhat prohibitive. Dreams often contain material that is nonsensical and challenging to interpret rationally, making the characterization of dreams from an objective point of view a perplexing task.

Some people rarely remember their dreams and erroneously conclude that they do not dream at all, while others experience vivid dreams with rich visual imagery and emotional content. Sometimes, the story lines that make up people's dreams follow a tight narrative and have a relatively smooth transition from a scene to scene, while at other times dreams appear as illogical and haphazard associations lacking a coherent sense of flow. Some people have full control of their dreams, exerting conscious control over the supposedly random events, which typify dreaming $[85,86]$, while others are mere bystanders watching the events unfold without any sense of agency approximating waking volition. With the multiplicity of dream dynamics, it is no surprise that there are differing from views on the nature of dreams, as a researcher's views on dreaming could be directly related to their own subjective experience $[87,88]$.

However, according to Tononi and Edelman [71], as has been mentioned before, dreams fulfill the minimum number of elements of conscious experience: conscious experience is integrated [each conscious scene is unified] and at the same time, it is highly differentiated [within a short time, one can experience any of a huge number of different conscious states]. Then, it could be said that dreaming is a type of conscious without awareness. Taking in account these two premises and the persistence of this mental activity in evolution [the inference of mental activity in some animals and the empirical observation of the existence of REM sleep in all the mammals that are detected with self-recognition, as dolphins, elephants and non-human primates, support that]. Other species cannot provide information about mental processes during sleep, so the controversy about whether some animals are dreaming during REM sleep persists. One perspective is that animals such as cats, which display threatinduced posturing and appear startled by invisible objects while in REM sleep, have confirmed that they are dreaming specially when they develop REM sleep behavior disorder after lesions in the Locus Coeruleus [86, 87].

One of the first and most important findings in the history of research on sleep and dreaming is that in which that produce relates of the phenomenon of dreaming and the physiological occurrence of rapid eye movement [REM] sleep [87]. While dreaming refers to "the subjective conscious experiences that we have during sleep" [88], REM sleep is a physiological stage of sleep. It has been established that dreaming does occur during REM sleep through the collection of dream reports from subjects awoken from REM sleep, though the same is true for Non-REM sleep [88, 90]. 
Rather than being a static process, sleep contains a number of discrete states defined by various physiological measures.

However, it remains to be elucidated whether the eyes scan dream images, despite studies that have retrospectively compared the direction of rapid eye movements to the dream recall recorded after having awakened the sleeper. In a recent report, researcher used the model of rapid eye movement sleep behavior disorder [when patients enact their dreams by persistence of muscle tone] to determine directly whether the eyes move in the same directions as the head and limbs. When rapid eye movements accompanied goal-oriented motor behavior during rapid eye movement sleep behavior disorder [e.g., grabbing a fictive object, hand greetings, climbing a ladder], which happened in 19 sequences, $82 \%$ were directed towards the action of the patient [same plane and direction]. When restricted to the determinant rapid eye movements, the concordance increased to $90 \%$. Rapid eye movements were absent in $38-42 \%$ of behaviors. This directional coherence between limbs, head and eye movements during rapid eye movement sleep behavior disorder suggests that, when present, rapid eye movements imitate the scanning of the dream scene. Since the rapid eye movements are similar in subjects with and without rapid eye movement sleep behavior disorder, this concordance can be extended to normal rapid eye movement sleep [90].

The last report supports our proposal that REM sleep in ASC without self-awareness o without any reflexive participation of what is going on. That is possible because prefrontal areas are not active in REM sleep, and as dreamers we believe everything in our dream. This lack of awareness is also connected to the total absence of critic to the situation that we are dreaming.

\section{THEORIES OF DREAMING}

The theory of dreaming most generally accepted, which proposed explains dreaming based on the physiology of REM sleep, by Hobson and McCarley's activation-synthesis hypothesis [55]. According to this hypothesis, dreams are the result of the forebrain responding to random activity, which starts in the brainstem. This is demonstrated by the pontogeniculate-occipital waves or PGO waves that occur during REM sleep. Specifically, the PGO, which is the area through which sensory information passes; and occipital areas, where visual information is processed. This random activity, emanates from the Pons, passes through similar sensory-relay stations as information from the environment, and is interpreted in a way that such stimuli leads to the phenomenology of dreaming [50, 51].

Although dreams tend to be rather bizarre, they are certainly not as disjointed as would be the case if this hypothesis were unilaterally true. Large samples of dream reports from a numerous studies point toward the fact that individuals see the majority of dreams as realistic and containing a connected storyline $[87,88,89,90]$. Likewise, to be discussed below, certain information is differentially represented in dreams [86].

Additional neuropsychological evidence reveals that the brainstem mechanism, which is a key ingredient in activation-synthesis theory, is not necessary for generated dreams. Some evidences [88] points towards the forebrain region as being crucial in the generation of dreams. If there is a reason to believe that dreaming is not just the random processing of information, but instead there is some pattern to the types of themes present in dreams and the possibility that dreams can consist of cohesive story-lines, then it seems logical to investigate why these patterns exist and what purpose they serve.

\section{MENTAL REHEARSAL}

It can be assumed that the brain is optimally designed to process information about the "real-world" and specifically for other human's beings. Sensory is the main source of that information. If the person rehearsal in a dream, we can react in an appropriate manner when confronted with environmental stimuli. Despite this fact, a large portion of mental life consists not of the processes of actual information, but rather the rehearsal of what to do when we come across stimuli from the environment, a specially with another human beings $[90,91]$. This rehearsal and the cognitive skills involved are likely to have a strong adaptive value.

\section{THREAT-SIMULATION THEORY}

To evaluate the threat simulation theory of dreaming, it is useful to discuss it in an evolutionary context, and consider whether dreaming meets the necessary requirements of evolution by natural selection; namely, genetic variation, inheritance, and differential fitness. As for the first condition, there is evidence that REM sleep is genetically varied between and within species. REM sleep seems to be exclusive to placental and marsupial mammals. This suggests a particular phylogeny of dreaming, and that there was some moment in which this characteristic was acquired and further spread to evolving species. Also, the amount of REM sleep placental and marsupial animals tend to require varies in a shared manner throughout their life cycle [90], pointing towards an underlying genetic control over dreaming.

When considering the third proposition of the differential fitness of dreaming in modern humans, it is important to understand the environment in which selection was occurring. Our human ancestors faced a number of challenges posed by interactions with nonspecific within and between groups [91], as well as some pressure in obtained food and protecting themselves from predators. In this environment, the ability to react when a real threat is apparent would obviously confer a survival advantage. Evidence from mental imagery and dream studies suggest that rehearsal in the dream is red as a real threat and, therefore, those individuals with these imagery skills to rehearse threatening scenarios should have an improved ability to deal with the threat, making them more likely to be the progenitors of offspring's. Through the survival and procreation of their offspring, this ability of, and propensity towards, imagery would be differentially passed on to future generations.

Dream content is biased toward negative elements reflecting threat, as opposed to positive elements. Data collected from over 500 dream reports by Hall and Van de Castle [1966] [87] indicated that about $80 \%$ contained negative emotions, while only about $20 \%$ contained positive emotions. These negative dreams are also in disproportion issues likely to contain threatening elements such as animals and strange males threatening. The evidence points towards the overrepresentation of threatening events in dreams, which should not occur if dream content in random. Through appropriating and learning to deal with these threats in dreams, 
it is proposed here that an animal could increase its overall evolutionary fitness. Posttraumatic Stress Disorder is overall the best example of the participation of REM sleep and other stress related mechanisms. People with PTSD may have alterations in brain regions central to the neurobiological fear response, specifically the amygdale and the hippocampus. The emotive aspects of dreams are relevant, in particular in the problem of remembering and the impact on the following day. The role of stress both in dream content and in memory is relevant. Some researchers proposed that dreams reflect a biological process of long-term memory consolidation with older memories [90].

\section{EPISTEMOLOGICAL CONSIDERATIONS ABOUT THE DREAM EXPERIENCE}

Dream could be considering experiences, the kinds that are described to at the self-level or first person experiences. In addition, those experiences happen in a particular world. That is, in fact, one of the many definitions of dreams: the presence of a world on the level of subjective experience while the subject is sleeping. The same definition could be directed to the waking experience. Because our experiences of the external world are mediated by sensory organs and the previous experiences [biography and development].

The main differences between dream and wakefulness experiences, is that first one, there is an absence of external sensorial information and awareness of dreaming. However, that is not absolute. There is always some information that can reach cognition when sleep occurs, and the subjective contamination of dream experiences [i.e., an alarm clock that is a fire alarm in dreams]. The latency from sleep to wakefulness is shorter for meaningful stimuli than nonmeaningful. Like hearing own names, or hearing other names, for example [92].

That the key element for these and other aspects related to this issue is the Thalamic - Cortical activities during sleep. Waking state is characterized by low amplitude, high frequency EEG waves, which are representative of the waking state. Sensorial information is able to pass because the low-level threshold of the cells firing in tonic mode [68]. When thalamic-cortical neurons became hyperpolarized, a large amplitude low frequency waves associated with delta sleep, produce a powerful inhibition that prevents inputs from peripheral areas of the nervous system. Auditory system could be an exception, although the signals are attenuated and completely block in REM sleep, because there are a high PGO density. In fact, animals with auditory stimulation during REM sleep, they have evoked potentials in cortical areas, but that happens when no PGO activity is coexistent $[93,94]$.

Some dreamers can induce more consciousness in a dream, which is called "Lucid Dream" [86, 87, 94, 95]. The beginning of this model of dream experiences was with red lights at REM sleep. That was a cue in which the dreamer knew that he was inside a dream. Later, most lucid dreamers developed some internal signals that were equivalent to the light turnin-on signals.

\section{SLEEP CONTINUITY}

Output blockade. There is a blockade of the ability to generate motor output. During REM sleep the atonia or func- tional paralysis is developed. That is the result of hyper polarization of alpha motoneurons in the medulla. There are, as has been mentioned before, some exceptions: extrinsic muscles of the eyes and some facial muscles that produce twitches. People suffering from REM sleep behavioral disorder, may have some anatomical problems that produce movements during REM sleep [i.e., lack of atonia]. This is a parasomnia that may overlap and status of dissociation. The recordings at the sleep laboratory showed excess of muscle tone on phasic EMG twice the activity during motor parasomnia. Since few seconds before REM sleep started. Michel Jouvet el al. [96] found a field wave, type of these PGO waves that were named heraldic, because they were announcing that REM sleep was about to start. The PGO waves use the same pathways that normal information during wake time is travel, so by this internal saturation, that may be another mechanism of a blockade.

There is not the possibility to control the attention inside the dream content. Dream experience is also described in some pathological situation, as in organic delirium [intoxication] some clinicians, in fact, describe that as "dreamlike" states. The series of bizarre dreams, could resemble some dysfunctional aspects that are observed in neurology in patients with some cortical injures. The misidentification of dream characters, even that she or he looked the opposite of the real person. This is named in clinic settings Frégoli-like phenomena. That, in the dream, could indicate that the cortical areas responsible for face recognition are not connected yet. In addition, some well-defined black and white dreams (achromatopsia). Dreams are like psychotic experiences. They are psychotic especially because when we are in this special world, we believe whatever happens as reality. Cognitive deficit, as well as memory and other aspects are related to a functional disconnection and timing in which some structures are first connected to others. As in waking, the worlds that we experience, all of them are model processes, but because they are experienced as "transparent we did not question its validity." Dreams are accepted as real. One of the first differences about reality in dreams and the one that we have when awake is that we are able to have a critic about what are going on and if possible to change it.

\section{CONCLUSIONS}

Conscious stop during sleep stages and it is ASC what replace it. Non-REM sleep is probably the only physiological period in which there is a complete disconnection of our brain, although as mentioned before that interruption is in module forms in which some areas are isolated from others. During REM sleep, also there is a kind of disconnection but with a different pattern. That is the functional separation of some areas of frontal lobes; probably orbit frontal areas that have been seen with low activity, while occipital are completely lit up. That could explain that dream without judgment as a primary kind of thinking or psychotic thoughts during dream. The probability for to have an autoscopy and OBS experiences may have a variability, so studies in this direction could give us more information about brain functioning and cognition. The inclusion of ASC and some processing of auditory information may contribute to the consciousness state when people are fully alert. Qualia is prominent during sleep and sometime detached of the usual object that we linked such quality. That, in fact is relevant because 
this process is storage and process in different brain structures. Broad definitions of factors that may contribute to consciousness, cognitive process as attention and memory, as well as new tools and translational approaches could give some lighter into this fascinating field.

In operative terms I can say that from neurobiological position, to be conscious requires both anatomical connectivity and oscillatory rhythms between them. As part of that, integration in fast way between different modules in cortex, specially the associative areas that must be centered in selfawareness region in the right parietal-occipital zone.

Consciousness fade out in Non-REM sleep because de cortex modules are fragmented, which means that for periods of sleep there is no connection, between cortical areas??

The essence of cortical operation is that, whatever computational takes place; other parts of the cortex get informed about it. That is possible due to intermediate a long range connections and oscillatory coupling mechanisms. Nevertheless, other type of structures inside the encephalon as cerebellum and basal ganglia, which almost the same degree of complexity as brain cortex, almost completely shut down, so why is necessary that the brain cortex has to be disconnected in delta sleep is one of the main questions to answered in the future ??.

In a second review paper about sleep deprivation effects I will try to figured out a hypothesis about that.

\section{REFERENCES}

[1] Zeman A. Consciousness. Brain 2001; 124: 1263-89.

[2] Cicogna P. Dreaming during sleep onset and awakening. Percept Mot Skills 1994; 78: 1041-42.

[3] Cicogna P, Bosinelli CM. Consciousness during dreams. Conscious Cogn 2001; 10: 26-41.

[4] Frith C. Attention to action and awareness of other minds. Conscious Cogn 2002; 11: 481-87.

[5] Frith C. How hallucinations make themselves heard? Neuron 1999; 22: 414-41.

[6] Sahraie A, Weiskrantz L, Barbur JL, Simmons A, Williams SC, Brammer MJ. Pattern of neuronal activity associated with conscious and unconscious processing of visual signals. Proc Natl Acad Sci USA 1997; 94: 9406-11.

[7] Sahraie A, Macleod MJ, Trevethan CT, et al. Improved detection following Neuro-Eye Therapy in patients with post-geniculate brain damage. Exp Brain Res 2010; 206: 25-34.

[8] Bosinelli M. Mind and consciousness during sleep. Behav Brain Res 1995; 69: 195-201.

[9] Jones BE, Mechanisms of Sleep-Wake States, In: Kryger MH, Roth T, Dement WC. $4^{\text {th }}$ ed. In Basic. Principles and Practice of Sleep Medicine. Ed. Philadelphia Elsevier Saunders, 2006; 136-153.

[10] Muzur A, Pace-Schott EF, Hobson JA. The prefrontal cortex in sleep. Trends Cogn Sci 2002; 6: 475-481.

[11] Broughton R. Human consciousness and sleep/waking rhythms: a review and some neuropsychological considerations. J Clin Neuropsychol 1982; 4: 193-218.

[12] Cheyne JA, Girard TA. Spatial characteristics of hallucinations associated with sleep paralysis. Cognit Neuropsychiatry 2004; 9: 281-300.

[13] Cheyne J A, Rueffer SD, Newby-Clark IR. Hypnagogic and hypnopompic hallucinations during sleep paralysis: neurological and cultural construction of the nightmare. Conscious Cogn 1999; 8: 319-37.

[14] Maquet PC, Phillips C. Functional brain imaging of human sleep. J Sleep Res 1998; 7(Suppl): 42-47.
[15] Schwartz S, Maquet P. Sleep, imaging and the neuro-psychological assessment of dreams. Trends Cogn Sci 2002; 6: 23-30

[16] Devinsky O, Feldmann E, Burrowes K, Bromfield E. Autoscopic phenomena with seizures. Arch Neurol 1989; 46: 1088.

[17] Alvaro LC. Hallucinations and pathological visual perceptions in Maupassant's fantastical short stories--a neurological approach. J Hist Neurosci 2005; 142: 100-115.

[18] Guilleminault C, Billiard M, Montplaisir J, Dement WC. Altered states of consciousness in disorders of daytime sleepiness. J Neurol Sci $1975 ; 26: 377-393$

[19] Hobson JA, Hoffman SA, Helfand R, Kostner D. Dream bizarreness and the activation-synthesis hypothesis. Hum Neurobiol 1987; 6: $157-164$.

[20] Saengpattrachai M, Sharma R, Hunjan A, et al., Nonconvulsive seizures in the pediatric intensive care unit: etiology, EEG, and brain imaging findings. Epilepsia 2006; 47: 1510-18.

[21] Gurstelle EB, de Oliveira JL. Daytime parahypnagogia: a state of consciousness that occurs when we almost fall asleep. Med Hypotheses 2004; 62: 166-18.

[22] Rainville P, Price DD. Hypnosis phenomenology and the neurobiology of consciousness. Int J Clin Exp Hypn 2003: 51: 105-129.

[23] Brown RJ. The cognitive psychology of dissociative states. Cognit Neuropsychiatry 2002; 7: 221-235.

[24] Nofzinger EA. Functional neuroimaging of sleep. Semin Neurol 2005; 25: 9-18

[25] Tononi G. Consciousness, information integration, and the brain. Prog Brain Res 2005; 150: 109-26.

[26] Lee L, Harrison LM, Mechelli A. A report of the functional connectivity workshop, Dusseldorf 2002. Neuroimage 2003; 19: 457465.

[27] Stickgold R. Neuroscience: a memory boost while you sleep. Nature 2006; 444: 559-60.

[28] Tononi G. An information integration theory of consciousness. BMC Neurosci 2006; 5: 42.

[29] Tononi G, Koch C. The neural correlates of consciousness: an update. Ann NY Acad Sci 2008; 1124: 239-261.

[30] Nir Y, Tononi G. Dreaming and the brain: from phe-nomenology to neurophysiology. Trends Cogn Sci 2010; 14: 88-100.

[31] Massimini M, Ferrarelli F, Huber R, Esser SK, Singh H, Tononi G. Breakdown of cortical effective connectivity during sleep. Science 2005; 30; 309: 2228-2232.

[32] Jones CB, Dorrian J, Jay SM, Lamond N, Ferguson S, Dawson D. Self-awareness of impairment and the decision to drier after an extended period of wakefulness. Chronobiol Int 2006; 23: 1253-1263.

[33] Reinoso-Suarez F, de Andres I, Rodrigo-Angulo ML, Garzon M. Brain structures and mechanisms involved in the generation of REM sleep. Sleep Med Rev 2001; 5: 63-77.

[34] Steriade M, Contreras D. Relations between cortical and thalamic cellular events during transition from sleep patterns to paroxysmal activity. J Neurosci 1995: 15: 623-42.

[35] Neider M, Pace-Schott EF, Forselius E, Pittman B, Morgan PT. Lucid dreaming and ventromedial versus dorsolateral prefrontal task performance. Conscious Cogn 2010 [Epub ahead of print].

[36] Dening TR, Berrios GE. Autoscopic phenomena. J Psychiatry 1994; 165: 808-17.

[37] Boussaoud D. The planning of action: can one separate attention from intention? Med Sci (Paris) 2003;19: 583-91.

[38] Shibasaki HM, Hallett M. What is the Bereitschaftspotential? Clin Neurophysiol 2006; 117: 2341-56.

[39] Beitman BD, Nair J, Viamonte GI. Why self-awareness? In: Neural Circuits for Self-Awareness. Self-Awareness Deficits in Psychiatric Patients. Beitman BD, Nair J, Eds. $1^{\text {st }}$ ed. Norton \& Company Inc., New York 2004; pp. 3-23.

[40] Viamontes GJ, Beitman BD, Viamontes CT, Viamontes JA. In: Neural Circuits for Self-Awareness. Self-Awareness Deficits in Psychiatric Patients. Beitman BD, Nair J, Eds. $1^{\text {st }}$ ed. Norton \& Company Inc., New York 2004; pp. 24-111. 
[41] Vignal JP, Maillard, L, McGonigal, A, Chauvel P. The dreamy state: hallucinations of autobiographic memory evoked by temporal lobe stimulations and seizures. Brain 2007; 130: 88-99.

[42] Nöe A. What does change blindness teach us about consciousness? Trends Cogn Sci 2005; 9: 218.

[43] Blakemore SJ, Smith J, Steel R, Johnstone CE, Frith CD. The perception of self-produced sensory stimuli in patients with auditory hallucinations and passivity experiences: evidence for a breakdown in self-monitoring. Psychol Med 2000; 30: 1131-1139.

[44] Blakemore SJ, Frith C. Self-awareness and action. Cur Opin Neurobiol 2003; 13: 219-224.

[45] Decety, J, Jackson PL. The functional architecture of human empathy. Behav Cogn Neurosci Rev 2004; 3: 71-100.

[46] Jeannerod M, Frak V. Mental imaging of motor activity in humans. Curr Opin Neurobiol 1999; 9: 735-9.

[47] Naito E, Kochiyama T, Kitada R, et al. Internally simulated movement sensations during motor imagery activate cortical motor areas and the cerebellum. J Neurosci 2002; 22: 3683-91.

[48] Nyberg L, Petersson KM, Nilsson LG, Sandblom J, Aberg C, Ingvar M. Reactivation of motor brain areas during explicit memory for actions. Neuroimage 2001; 14: 521-28.

[49] Stuss DT. A sensible approach to mild traumatic brain injury. Neurology 1995; 45: 1251-52.

[50] Thomas AK, Loftus EF. Creating bizarre false memories through imagination. Mem Cogni 2002; 30: 423-31.

[51] Tsai YT, Chan HL, Lee ST, Tu PH, Chang BL, Wu T. Significant thalamocortical coherence of sleep spindle, theta, delta, and slow oscillations in NREM sleep: Recordings from the human thalamus. Neurosci Lett 2010; 485: 173-7.

[52] Issa EN, Wang X. Sensory Responses during Sleep in Primate Primary and Secondary Auditory Cortex. J Neurosci 2008; 28: 14467-80.

[53] Vassalli A, Dijk DJ. Sleep function: current questions and new approaches. Eur J Neurosci 2009; 29: 1830-41.

[54] McCarley RW, Massaquoi SG. Neurobiological structure of the revised limit cycle reciprocal interaction model of REM cycle control. J Sleep Res 1992; 1: 132-7.

[55] McCarley RW. REM sleep and depression: common neurobiological control mechanisms. Am J Psychiatry 1982; 139: 565-70.

[56] McGinty DJ, Drucker-Colin RR, Morrison A, Permeggiani PL. Brain Mechanisms of Sleep . Sleep mechanisms: biology and control of REM sleep. 1985; Raven Press New York, 1-31.

[57] Tononi G, Massimini M. Why does consciousness fade in early sleep? Ann NY Acad Sci 2008; 1129: 330-334.

[58] Laufs H, Holt JL, Elfont R, et al. Where the BOLD signal goes when alpha EEG leaves. Neuroimage 2006; 31: 1408-18.

[59] Olbrich S, Mulert C, Karch S, et al. EEG-vigilance and BOLD effect during simultaneous EEG/fMRI measurement. Neuroimage 2009; 45: 319-32.

[60] Boly M, Phillips C, Tshibanda L, Vanhaudenhuyse A, Schabus M, $\mathrm{Ng}-\mathrm{Vu} \mathrm{TT}$, et al. Intrinsic brain activity in altered states of consciousness: how conscious is the default mode of brain function? Ann N Y Acad Sci 2008; 1129: 119-129.

[61] Fukunaga M, Horovitz SG, de Zwart JA, et al. Metabolic origin of BOLD signal fluctuations in the absence of stimuli. J Cereb Blood Flow Metab 2008; 28: 1377-1387.

[62] Laufs H, Holt JL, Elfont R, et al. Where the BOLD signal goes when alpha EEG leaves. Neuroimage 2006; 31: 1408-1418.

[63] Steriade M. Acetylcholine systems and rhythmic activities during the waking--sleep cycle. Prog Brain Res 2004; 145: 179-196.

[64] Steriade M. Central core modulation of spontaneous oscillations and sensory transmission in thalamocortical systems. Curr Opin Neurobiol 1993; 3: 619-625.

[65] Steriade M, Nunez A, Amzica F. A novel slow [<1 Hz] oscillation of neocortical neurons in vivo: depolarizing and hyperpolarizing components. J Neurosci 1993; 13: 3252-3265.

[66] Steriade M, Dossi R, Curro, Contreras D. Electrophysiological properties of intralaminar thalamocortical cells discharging rhythmic [approximately $40 \mathrm{HZ}$ ] spike-bursts at approximately $1000 \mathrm{HZ}$ during waking and rapid eye movement sleep. Neuroscience 1993; 56: 1-9.

[67] Steriade M, Contreras D, Curro DR, Nunez A. The slow $[<1 \mathrm{~Hz}]$ oscillation in reticular thalamic and thalamocortical neurons: scenario of sleep rhythm generation in interacting thalamic and neocortical networks. J Neurosci 1993; 13: 3284-3299.

[68] Steriade M, Amzica F, Nunez A. Cholinergic and noradrenergic modulation of the slow [approximately $0.3 \mathrm{~Hz}$ ] oscillation in neocortical cells. J Neurophysiol 1993; 701: 385-400.

[69] Steriade M, Contreras D, Amzica F, Tomofeev I. Synchronization of fast activities in brain stem cholinergic in intrathalamic and thalamocorical systems. J Neurosci 1996; 16: 2788-800.

[70] Steriade M, Nunez A, Amzica F. Intracellular analysis of relations between the slow $[<1 \mathrm{~Hz}]$ neocortical oscillation and other sleep rhythms of the electroencephalogram. J Neurosci 1993; 13: 326683

[71] Tononi G, Edelman GM. Consciousness and complexity. Science 1998; 282: 1846-51.

[72] Buzsaki G. The brain's default state: Self Organized Oscillations in The Rest and Sleep. In : Rhythms of the Brain. Oxford University Press. New York. 2006; pp. 175-205.

[73] Buzsaki G. The Gamma Buzz: Gluing by Oscillations in the Waking Brain In : Rhythms of the Brain. Oxford University Press. New York. 2006; pp. 231-61.

[74] Ogawa T. Characterization of developmental changes in EEGgamma band activity during childhood using the autoregressive model. Acta Paediatr Jpn 1998; 40: 446-52.

[75] Kaufmann CR, Wehrle R, Wetter TC, et al. Brain activation and hypothalamic functional connectivity during human non-rapid eye movement sleep: an EEG/fMRI study. Brain 2006; 129: 655-67.

[76] Lenggenhager B, TadiT, Mezinger T, Video Ergo Sum. Manipulating bodily self-consciousness. Science 2007; 317: 1096-99.

[77] Ehrsson E. The Experimental Induction of Out-of-Body Experiences. Science 2007; 317: 1048 .

[78] Ridder DD, Laere KV. DuPont P, Menovsky T, Heyning PV. Visualizing out-of-body experience in the brain. NJM 2007; 357: 182933.

[79] Brugger P. Reflective mirrors: perspective taking in autoscopic phenomena. Cogn Neuropsychiatry 2002; 7: 179-194.

[80] Gabbard GO, Twemlow SW, Jones FC. Differential diagnosis of altered mind/body perception. Psychiatry 1982; 45: 361-69.

[81] Bonanni E, Cipolli C, Ludice A, Mazzetti M, Murri L. Dream recall frequency in epilepsy patients with partial and generalized seizures: a dream diary study. Epilepsia 2002; 43: 889-95.

[82] Landau ME, Maldonado JY, Jabbari B. The effects of isolated brainstem lesions on human REM sleep. Sleep Med 2005; 6: 37-40.

[83] Taber K, Hurley RA. Functional neuroanatomies of sleep and sleep deprivation. J Neuropsychiatry Clin Neurosci 2006; 18: 1-5.

[84] Fell J, Fernandez G, Klaver P, et al. Rhinal-hippocampal coupling during declarative memory formation: dependence on item characteristics. Neurosci Lett 2006; 407: 37-41.

[85] LaBerge S, Levitan L, Dement WC. Lucid dreaming: Physiological correlates of consciousness during REM sleep. J Mind Behav 1986; 7: $251-8$

[86] La Berge SP. Lucid dreaming as a learnable skill: A case study. Percep Motor Skills 1980; 51: 1039-42.

[87] LaBerge S. Behavioural and Brain Sciences. Lucid dreaming: Evidence and methodology. Behav Brain Sci 2000; 23: 962-964.

[88] Solms M. Dreaming and REM sleep are controlled by different brain mechanisms. Behavioral and Brain Sciences Cambridge University Press, Cambridge MA 2000; 23: 843-850.

[89] Hartmann E, Zborowsky M, Kunzendorf R. The Emotion pictured by a dream: AM examination of emotions contextualized in dreams. Sleep Hypnosis 2001; 3: 33-43.

[90] Hobson JA, Dreaming: Introduction to the science of sleep. Oxford University Press 2002

[91] Siegel, J. REM Sleep. In: Principles and Practice of Sleep Medicine. Kryger M, Roth T and Dement WC. 4th ed. Philadelphia: Elsevier Saunders 2005; 120-19. 
[92] Oswald I, Taylor AM, Treisman M. Discriminative responses to stimulation during human sleep. Brain 1960; 83: 440-453.

[93] Salin-Pascual RJ, Granados-Fuentes D, de la Fuente JR, DruckerColin R. Effects of auditory stimulation during rapid eye movement sleep in healthy volunteers and depressed patients. Psychiatry Res1991; 38: 237-246.

[94] Dennet D. Conscious explained. Back Bay Books, New York 1991; $1-65$.
[95] LaBerege S. Psychophysiogical Studies of Consciousness during REM Sleep in RR. Bootzen, J.F. Kihlstrom and DL, Schacter Eds. Sleep and Cognition. American Psychological Association.1990; 109-126.

[96] Jouvet M. The mechanism of waking: from mesencephalic reticular system to multiple networks. Arch Physiol Biochem1996; 104: 762.

Received: December 26, 2011

Revised: April 17, 2011

Accepted: April 18, 2011

(C) Rafael J. Salín-Pascual; Licensee Bentham Open.

This is an open access article licensed under the terms of the Creative Commons Attribution Non-Commercial License (http://creativecommons.org/licenses/by-nc/3.0/) which permits unrestricted, non-commercial use, distribution and reproduction in any medium, provided the work is properly cited. 